\title{
Médiévales
}

Langues, Textes, Histoire

71 | automne 2016

Conflits et concurrence de normes

\section{Les voix du peuple à la fin du Moyen Âge}

The voices of the people in the late Middle Ages

\section{Didier Lett}

\section{(2) OpenEdition}

Journals

Édition électronique

URL : https://journals.openedition.org/medievales/7931

DOI : 10.4000/medievales.7931

ISSN : $1777-5892$

Éditeur

Presses universitaires de Vincennes

Édition imprimée

Date de publication : 20 novembre 2016

Pagination : 159-176

ISBN : 978-2-84292-565-9

ISSN : 0751-2708

Référence électronique

Didier Lett, «Les voix du peuple à la fin du Moyen Âge », Médiévales [En ligne], 71 | automne 2016, mis en ligne le 20 novembre 2018, consulté le 29 avril 2022. URL : http://journals.openedition.org/ medievales/7931; DOI : https://doi.org/10.4000/medievales.7931 


\section{Didier Lett}

\section{Les voix du peuple à la fin du Moyen Âge}

Comme leurs titres l'indiquent, les trois ouvrages qui font l'objet de ce «point de vue » ont en commun de s'intéresser aux groupes qui occupent la partie «basse » du corps social à la fin du Moyen Âge pour tenter d'écouter leurs voix ${ }^{1}$. Les manifestations sociales et politiques des « dominés » sont étudiées par Luigi Provero dans le centre et le sud du Piémont (vallée de Suse, Montferrat, Tortonese et région de Cuneo au XIII ${ }^{\mathrm{e}}$ siècle), par Laure Verdon en Provence (basse vallée du Rhône, baillie de Castellane et viguerie de Puget-Théniers dans la seconde moitié du $\mathrm{XIII}^{\mathrm{e}}$ siècle et au début du XIV ${ }^{\mathrm{e}}$ siècle) et, pour l'ouvrage collectif (par la suite nommé The Voices of the People) de l'Angleterre à la Castille, en passant par les Flandres, le Languedoc et les villes italiennes du XIV siècle au $\mathrm{XVI}^{\mathrm{e}}$ siècle. Les ouvrages sont rédigés dans trois langues différentes (italien, français et anglais), s'inscrivant dans des traditions historiographiques diverses. En France, l'histoire des humbles a été longtemps marquée par l'influence d'une histoire catholique et par l'anthropologie historique. En Italie, le popolo a été étudié dans le cadre de l'histoire communale, comme groupe politique (les cives) et comme groupe social (les pedites, ceux qui

1. L. Provero, Le Parole dei sudditi. Azioni e scritture della politica contadina nel Duecento, Spolète, 2012 ; L. Verdon, La Voix des dominés. Communautés et seigneurie en Provence au bas Moyen Âge, Rennes, 2012 ; et J. Dumolyn, J. Haemers, H. R. Oliva Herrer et V. Challet éd., The Voices of the People in Late Medieval Europe. Communication and Popular Politics, Turnhout, 2014. Le livre de Laure Verdon est issu d'une Habilitation à diriger des recherches (HDR) soutenue en 2007. L'ouvrage collectif en anglais est le résultat écrit de trois projets de recherche, belge et espagnols, menés durant cinq années, intitulés City and Society in the Low Countries (c. 1200-c. 1850). The 'condition urbaine' : between résilience and vulnerability; Espacio público, opinión y comunicación política a fines de la Edad Media ; El poder de la comunidad? : Lenguaje y practicas políticas populares a fines de la Edad Media. 
ne vont pas à cheval) ${ }^{2}$. Quant à l'ouvrage collectif, il s'insère dans des usages historiographiques plus disparates où domine cependant la tradition méthodologique anglophone. Mais ces trois livres ont en commun d'être produits dans un paysage historiographique aujourd'hui profondément renouvelé : ils bénéficient des apports les plus récents d'études sur l'oralité et la critique de la « voix vive » dans les sources inquisitoires qui aident à mieux décrypter les manifestations orales des gens du peuple ${ }^{3}$, de l'essor des travaux sur la communication politique, l'information et la capacité d'organisation collective des paysans permettant de reconsidérer les modes d'expressions populaires au sein des communautés ${ }^{4}$, du développement d'une nouvelle history from below qui doit toujours beaucoup aux travaux pionniers d'Edward P. Thomson ${ }^{5}$ et, enfin, de nouvelles approches sur les mouvements de contestation populaire ${ }^{6}$.

Après un détour par l'historiographie française relative au peuple afin de mieux mettre en valeur les apports fondamentaux de ces ouvrages, nous proposons de montrer comment le «peuple » est ici saisi dans sa globalité, comme une catégorie souple et plurielle, à travers son rapport au pouvoir et aux élites dans une dialectique où l'accent est mis sur sa

2. Comme le souligne François Menant, à cause de cette double acception et de cette ambiguïté, les historiens italiens, par convention, utilisent une graphie différente en fonction de ce qu'ils veulent indiquer : Popolo au sens d'organisation politique et popolo, pour désigner un groupe social (F. Menant, L'Italie des communes [1100-1350], Paris, 2005, p. 65).

3. Pour un bilan récent, on se reportera au dossier «L'oral et l'écrit », dans J.-C. SchmitT et O. G. EXLE éd., Les Tendances actuelles de l'histoire du Moyen Âge en France et en Allemagne, Paris, 2002, p. 127-169. Pour rester dans le domaine inquisitoire, documentation fortement convoquée dans ces trois ouvrages, voir D. LA CAPRA, « The Cheese and the Worms : the Cosmos of a Twentieth-Century Historian », dans EAD., History and Criticism, New York, 1985, p. 49-69 ; R. RosAlDo, « From the Door of his Tent. The Fieldworker and the Inquisitor », dans J. Clifford et G. Marcus éd., Writing Culture, Berkley/Los Angeles/ Londres, 1986, p. 77-97 ; J. ARnOLD, Inquisition and Power. Catharism and the Confessing Subject in Medieval Languedoc, Philadelphie, 2001 ; D. LAURENDEAU, "Cet évêque fait sortir la vérité, même si cela ne plaît pas à ceux qui la disent ". Faire parler et savoir taire au tribunal d'Inquisition de Pamiers (1318-1325), thèse de doctorat des universités de Paris 1 Panthéon-Sorbonne et du Québec de Montréal, dirigée par M. Bourin et M. Hébert, 2008 (dactyl.), en particulier p. 30-37 ; D. LETT, Un procès de canonisation au Moyen Âge. Essai d'histoire sociale. Nicolas de Tolentino, 1325, Paris, 2008, p. 139-155.

4. C. Wickham, Comunità e clientele nella Toscana del XII secolo. Le origini del comune rurale nella Piana di Lucca, Rome, 1995 ; trad. fr., Communautés et clientèles en Toscane au XII siècle. Les origines de la commune rurale dans la plaine de Lucques, Rennes, 2001. Pour une synthèse dans le monde paysan, voir L. Feller, Paysans et seigneurs au Moyen Âge, VIII ${ }^{e}$ XV siècles, Paris, 2007, chap. 7, p. 192-218.

5. Voir E. P. Thompson, Les Usages de la coutume. Traditions et résistances populaires en Angleterre, XVII $-X I X^{e}$ siècle, Paris, [1991] 2015, et la récente mise en point critique de S. Cerutti, «Who is below ? E. P. Thompson, historien des sociétés modernes : une relecture », Annales HSS, 70/4 (2015), p. 931-955.

6. Voir en dernier lieu : S. CoHn, Popular Protest in Late Medieval Europe : Italy, France, and Flanders, Manchester, 2004. 
subordination (dominés, sudditi), mais aussi sur ses capacités d'actions politiques. Ensuite, le but est bien d'entendre la voix du peuple (voices, voix, parole), c'est-à-dire les modes d'expression, de la soumission à la lutte ouverte en passant par le consentement, la négociation ou le refus passif. Enfin, révélateur de profonds changements de paradigme et de méthodes nouvelles d'investigation, il s'agit aussi d'une réflexion soutenue sur la documentation.

\section{La voix du peuple dans I'historiographie française}

L'historiographie française récente a bien davantage privilégié les élites (les rois, les princes, l'aristocratie, la noblesse, les chevaliers, les notables, etc.) ${ }^{7}$. Cependant, chercher à connaître la « culture populaire » et à déceler les manières dont les humbles s'expriment n'a jamais quitté l'horizon d'attente des historiens malgré l'évocation récurrente et l'excuse du «manque de sources ». L'intérêt pour les plus modestes est un phénomène ancien qui accompagne la naissance même de l'histoire en tant que science. Sans remonter à Michelet (1846), pour qui le peuple est un collectif et une allégorie de l'Histoire ${ }^{8}$, les historiens de la fin du XIX ${ }^{\mathrm{e}}$ siècle, dans un contexte de vif engouement pour la vie privée ${ }^{9}$, tendaient déjà l'oreille afin

7. Pour se limiter aux ouvrages collectifs en histoire médiévale, citons Les Élites urbaines au Moyen Âge, XXVII Congrès de la SHMESP (Rome, mai 1996), Paris, 1997 ; F. MenANT et J.-P. JESSENNE éd., Les Élites rurales dans l'Europe médiévale et moderne (XXVII ${ }^{\mathrm{es}}$ Journées internationales d'histoire de l'Abbaye de Flaran), Toulouse, 2007 ; F. BougARD, L. FELLER et R. LE JAN éd., Les Élites au haut Moyen Âge. Crises et renouvellements, Turnhout, 2006 ; J. P. Devroey, L. Feller et R. Le Jan éd., Les Élites et la richesse au haut Moyen Âge, Turnhout, 2010 ; F. Bougard, H.-W. Goetz, et R. LE JAN éd., Théories et pratiques des élites au haut Moyen Âge. Conception, perception et réalisation sociale / Theorie und Praxis frühmittelalterlichen Eliten. Konzepte, Wahrnehmung und soziale Umsetzung, Turnhout, 2011 ; L. Feller, M. Kaplan et C. Picard éd., Élites rurales méditerranéennes au Moyen Âge, dossier paru dans les Mélanges de l'École française de Rome - Moyen Âge, 124/2 (2012), [en ligne] : https://mefrm.revues.org/713 [consulté le 7 septembre 2016]. Face à cette écrasante domination des travaux sur les élites (qui ne se tarit pas), les études sur le peuple sont parfois présentées comme un « rééquilibrage » : les deux colloques organisés à Lille par l'équipe «Espaces et cultures de la ville » en 2000 et en 2001 (publiés en 2002 et 2003) se donnent explicitement pour but « de procéder à un rééquilibrage de la recherche en histoire sociale urbaine en conduisant aussi une réflexion sur les milieux qui vivent hors des groupes socialement et culturellement dominants » : voir P. GuIGNET, «Introduction », dans Le Peuple des villes dans l'Europe du Nord-Ouest (fin du Moyen Âge-1945), vol. 1, Lille, 2002, p. 6.

8. J. Michelet, Le Peuple, Paris, 1846.

9. Citons, comme emblématique, la monumentale fresque, en 27 volumes, réalisée par A. L.A. Franklin, La Vie privée d'autrefois : arts et métiers, modes, mours, usages des Parisiens, du XII ${ }^{e}$ au XVII' siècle d'après des documents originaux ou inédits, Paris, 1887-1902. 
de percevoir le murmure s'élevant de l'écrasante majorité de la population dans leur vie ordinaire. En 1882, Felix Brun écrivait ${ }^{10}$ :

Nous allons chercher ce que fut le paysan dans sa vie privée [...]. Visiter sa chaumière, inventorier son petit mobilier, jeter un coup d'œil sur son gardemanger, prendre part à ses fêtes et à ses délassements, reconstituer, après cela, non tout son rôle historique et social, mais un peu de sa physionomie ordinaire, de sa manière d'être de tous les jours.

L'année suivante, Clarisse Bader avouait son projet de pénétrer dans les foyers français du passé ${ }^{11}$ :

Dans cette partie de mon œuvre, j'interroge les personnes qui ont vécu dans ces trois siècles $\left[\mathrm{XVI}^{\mathrm{e}}-\mathrm{XVIII}{ }^{\mathrm{e}}\right.$ siècle], je recueille leurs témoignages, ces témoignages que nous livrent particulièrement les mémoires domestiques, les correspondances privées, tous les documents intimes auxquels notre époque attache justement un si grand prix.

Le souci d'étudier les classes populaires s'affirme et se concrétise pleinement dans la seconde moitié du $\mathrm{xx}^{\mathrm{e}}$ siècle. Les sources et les méthodes de la démographie historique ont permis en partie, surtout pour l'époque moderne, de réaliser cette ambition de reconstituer la vie des habitants d'un village ou d'une ville du berceau à la tombe. L'histoire marxiste ou marxisante des années 1960-1970 a éclairé la condition ouvrière. Pour le Moyen Âge, elle s'est davantage intéressée au mode de production féodal qu'aux conditions de vie des plus humbles ou aux mouvements hérétiques comme formes de contestation sociale ${ }^{12}$. À partir de ces mêmes années, la progressive démocratisation de l'entrée à l'Université, permettant à des gens d'extraction modeste de pénétrer le monde de la recherche, renforce l'intérêt pour les gens ordinaires ${ }^{13}$.

Citons, sans aucun souci d'exhaustivité bien entendu, quelques jalons significatifs et emblématiques de cette histoire. En 1958, Louis Chevalier, en proposant une histoire biologique et qualitative du crime, dévoile les souffrances des «classes laborieuses » durant la première moitié

10. F. Brun, La Vie privée des paysans au Moyen Âge et sous l'Ancien Régime, Paris, 1882, p. 5-6.

11. C. BADER, La Femme française dans les temps modernes, Paris, 1883, préface, p. v-VI.

12. Cette « croyance » dans les « hérésies populaires» se perçoit très nettement dans le fameux et pionnier ouvrage sous la direction de J. LE GofF, Hérésies et sociétés dans l'Europe préindustrielle, $X I^{e}-X V I I^{e}$ siècles, Paris/La Haye, 1968. Pour un bilan historiographique et l'évolution des historiens sur l'interprétation des hérésies, voir A. VAUCHEZ, « L'historiographie des hérésies médiévales », dans J. Revel et J.-C. Schmitт éd., L'Ogre historien. Autour de Jacques Le Goff, Paris, 1998, p. 243-258 ; à compléter par M. Zerner, « Hérésie », dans J. Le GoFf et J.-C. Sснмітт éd., Dictionnaire raisonné de l'Occident médiéval, Paris, 1998, p. 464-481.

13. Pierre Goubert se plaisait à rappeler qu'il était issu du monde des humbles dont il s'était fait l'historien. 
du XIX ${ }^{\mathrm{e}}$ siècle $^{14}$. En 1963, dans les Annales, François Furet propose de mieux définir les «classes inférieures » de l'époque moderne ${ }^{15}$. En 1966, Pierre Goubert, exploitant les registres paroissiaux, les inventaires après décès et les archives hospitalières, attire l'attention sur les vingt millions de Français qui ont aussi fait la France de Louis XIV ${ }^{16}$. Des historiens et des historiennes ont poursuivi inlassablement dans cette voie, telle Arlette Farge dont l'œuvre entière vise à exhumer les gens ordinaires et les plus démunis de Paris au $\mathrm{XVIII}^{\mathrm{e}}$ siècle, en auscultant leur silence, leur souffle et leurs voix ${ }^{17}$, ou Daniel Roche qui, après des premiers travaux consacrés aux élites, soucieux de saisir l'ensemble de la société de la fin de l'Ancien Régime, s'intéresse également aux diverses formes de la culture populaire ${ }^{18}$. Que l'on regarde donc du côté de la démographie historique ou de l'histoire sérielle, de l'histoire des mentalités ou de l'anthropologie historique, de la microstoria ou d'autres tentatives contemporaines de reconstitution biographique, on retrouve toujours cette volonté de reconstituer la vie des personnes « ordinaires » : un meunier, un sabotier, un compagnon-verrier, etc. ${ }^{19}$

Les médiévistes ne sont pas demeurés en marge de cette tendance historiographique. En 1981, Aaron J. Gourevitch se demandait ${ }^{20}$ :

Peut-on jamais espérer voir le monde médiéval non pas avec les yeux des auteurs érudits de cette époque, mais tel que le voyait l'homme ordinaire ? Autrement dit : est-il possible de connaître la culture populaire du Moyen Âge ?

Les grandes monographies d'histoire rurales ou urbaines des années 1970-1990 ont également centré leur regard sur les plus humbles et sur leur position dans les rapports de production, leurs conditions de vie, en montrant déjà combien les paysans avaient pu s'organiser politiquement et efficacement face à leur seigneur ${ }^{21}$. La part belle a été faite aux «pauvres »

14. L. Chevalier, Classes laborieuses et classes dangereuses à Paris pendant la première moitié du XIX siècle, Paris, 1958 (rééd. 2002).

15. F. Furet, «Pour une définition des classes inférieures à l'époque moderne », Annales ESC, 18/2 (1963), p. 459-474.

16. P. Goubert, Louis XIV et vingt millions de Français, Paris, 1966.

17. A. FARge, Vivre dans la rue à Paris au XVIII siècle, Paris, 1979 ; EAD., Essai pour une histoire des voix au dix-huitième siècle, Paris, 2009 et EAD., Le Silence, le souffle, Paris, 2008.

18. D. Roche, Le Peuple de Paris. Essai sur la culture populaire au XVIII siècle, Paris, 1981.

19. Le meunier Menocchio dans C. GinzBurg, Le Fromage et les vers. L'univers d'un meunier frioulan du XVI siècle, Paris, [1976] 1980 ; le compagnon-verrier Jacques-Louis Ménétra, dans D. Roche éd., Journal de ma vie : édition critique du journal de JacquesLouis Ménétra, compagnon vitrier au XVIII siècle, Paris, 1982 ; le sabotier dans A. CoRBIN, Le Monde retrouvé de Louis-François Pinagot, Sur les traces d'un inconnu (1798-1876), Paris, 1998.

20. A. J. Gourevitch, La Culture populaire au Moyen Âge. Simplices et docti, Paris, 1996, p. 10 .

21. Citons juste, dans ce domaine, M. Bourin et R. Durand, Vivre au village au Moyen Âge. Les solidarités paysannes du XI e au XIII siècle, Paris, 1984. 
et aux « marginaux ». Michel Mollat s'est intéressé aux plus démunis de la société et à l'assistance ${ }^{22}$, tandis que Bronislaw Geremek étudiait les « marginaux », terme appelé à une belle fortune au sein de l'anthropologie historique ${ }^{23}$. Les médiévistes ont également cherché à connaître la « culture populaire » - ou la « religion populaire » ou la « religion des laïcs » ou les religions des « incrédules », parfois encore nommée « culture folklorique »qui se serait opposée à la « culture savante $»^{24}$. Appartiennent à la religion populaire ce qui est élaboré par le peuple, ce qui est utilisé et vécu par lui, et ce qui lui est destiné ${ }^{25}$. Mais quel que soit le terme utilisé, les médiévistes qui ont travaillé sur ces aspects s'accordent pour reconnaître qu'entre religion officielle et religion populaire les contacts sont nombreux et la circulation des croyances constantes. Ce mouvement d'ailleurs ne se réalise pas seulement, comme le pensait Georges Duby, par « percolation sociale » (de haut en bas), mais s'avère aussi le résultat d'une dialectique complexe et d'échanges permanents et peut donc venir également d'en bas : ce sont nos sources qui donnent l'illusion d'un mouvement descendant. Voulant imiter les ethnologues, les médiévistes ont cru aussi qu'ils pouvaient « enregistrer » les voix du peuple, principalement à travers les sources inquisitoires. En 1975, Emmanuel Le Roy Ladurie était persuadé qu'au tournant du XIII et XIV ${ }^{\mathrm{e}}$ siècle, Jacques Fournier, à Montaillou, avait « donné la parole aux villageois, et même à tout un village en tant que tel ${ }^{26}$ ». La recherche de la manière dont le peuple s'est exprimé au Moyen Âge se retrouve enfin dans les travaux sur les révoltes populaires ou les tumultes ${ }^{27}$,

22. M. Mollat, Les Pauvres au Moyen Âge, Paris, 1978 ; ID. éd., Études sur l'histoire de la pauvreté (du Moyen Âge au XvI siècle), Paris, 1974. Voir aussi J.-L. GogLIN, Les Misérables dans l'Occident médiéval, Paris, 1976.

23. B. GeremeK, Les Marginauxparisiens aux XIVe et XV viècles, Paris, 1976 et J.-C. SchmitT, «L'histoire des marginaux », dans J. Le Goff éd., La Nouvelle Histoire, Paris, 1978 (rééd. 1988), p. 277-305. Voir aussi, pour l'aspect historiographique, A. FARGE, « Marginalités », dans C. Delacroix, F. Dosse, P. Garcia et N. Offenstadt éd., Historiographies. Concepts et débats, Paris, 2010, p. 491-501.

24. Opposition qui, me semble-t-il, a été davantage et beaucoup plus longtemps utilisée en histoire moderne. Pour l'époque médiévale, voir E. Delaruelle, La Piété populaire au Moyen Âge, Turin, 1975 ; J.-C. SchMitт, « Religion populaire et culture folklorique », Annales ESC, $31 / 5$ (1976), p. 941-953 ; G. LoBrichon, La Religion des lä̈cs en Occident, XI $I^{e}$-XV siècles, Paris, 1994 ; P. Golinelli, Il Medioevo degli incrudeli. Miscredenti, beffatori, anticlericali, Milan, 2009.

25. M. LAUWERS, «"Religion populaire", culture folklorique, mentalités. Notes pour une anthropologie culturelle du Moyen Âge », Revue d'histoire ecclésiastique, 82/2 (1987), p. 221-258 ; ID., « Religion populaire », dans Catholicisme, vol. 12, 1990, col 835-849.

26. E. Le Roy Ladurie, Montaillou, village occitan de 1294 à 1324, Paris, 1975, avantpropos, p. 9.

27. M. Mollat et P. WolfF, Ongles bleus, Jacques et Ciompi. Les révolutions populaires en Europe aux XIV et XVe siècles, Paris, 1970 ; H. NeVEux, Les Révoltes paysannes en Europe (XIV'-XVII siècle), Paris, 1997 ; A. Stella, La Révolte des Ciompi. Les hommes, les lieux, le travail, Paris, 1993. 
et dans ceux relatifs aux conditions de travail des gens ordinaires ${ }^{28}$. On peut considérer le colloque tenu à l'université de Montréal en 1999 sur le « petit peuple » comme l'aboutissement de ce mouvement de fond qui a animé la médiévistique au cours du $\mathrm{xx}^{\mathrm{e}}$ siècle, rencontre dont le but ambitieux était à la fois de faire le point sur le lexique utilisé pour nommer les plus humbles, de jauger la manière dont ils sont perçus dans les différents discours et de tenter de cerner leurs conditions « réelles » de vie et de travail ${ }^{29}$.

\section{Le peuple : une catégorie ou de multiples acteurs?}

Le peuple auquel s'intéressent les trois ouvrages est à prendre dans une acception étroite : non pas l'ensemble d'une société par opposition à d'autres peuples ${ }^{30}$, mais bien la partie inférieure de celle-ci, qui se distingue, parfois de manière très binaire, des élites. Il s'agit d'une catégorie exogène construite à partir de référents médiévaux tels que populus, plebs, vulgus, minores, laborantes, rustici, simplices, pauperes, nihiles, etc. : ceux qui ne possèdent pas ou peu, qui vivent modestement, dans l'incertitude, qui dégagent rarement du surplus, qui n'ont pas ou peu de considération, de renommée et de reconnaissance ${ }^{31}$, qui n'ont pas ou peu de pouvoir, pas ou peu d'instruction, qui n'accèdent pas à l'écrit, etc. Dans la conclusion du colloque de Montréal consacré au «petit peuple », Claude Gauvard proclamait : « Mais le peuple ne peut pas se réduire à ses marges ${ }^{32}$. » De fait, les gens du peuple abordés dans ces trois ouvrages ne se limitent plus aux « marginaux »d'antan, parce qu'ils occupent le centre de la société et représentent l'écrasante majorité des médiévaux.

Une catégorie est « un agrégat d'individus conçu et construit par le chercheur (ou défini par les représentations des contemporains) qui considère que des individus présentent, à un point de vue donné, des caractéristiques communes $^{33} »$. C'est un instrument utile pour ordonner la complexité sociale, pratique pour classer, mais souvent très arbitraire. Son utilisation

28. P. Braunstein, Travail et entreprise au Moyen Âge, Bruxelles, 2003 ; A. Stella éd., «Les dépendances au travail », dossier paru dans Médiévales, 30 (1996); P. BECK, P. BERNARDI et L. FELLER éd., Rémunérer le travail au Moyen Age : pour une histoire sociale du salariat, Paris, 2014.

29. P. Boglioni, R. Delort et C. Gauvard éd., Le Petit Peuple dans l'Occident médiéval. Terminologies, perceptions, réalités, Paris, 2002.

30. Gérard Fritz a posé la question de savoir si le peuple s'est pensé comme peuple avant le XVII ${ }^{\mathrm{e}}$ siècle : G. FrITZ, L'Idée de peuple en France du XVII au XIXe siècle, Strasbourg, 1988.

31. G. Todeschini, Aux pays des sans-nom. Gens de mauvaise vie, personnes suspectes ou ordinaires du Moyen Âge à l'époque moderne, Paris, [2007] 2015.

32. C. Gauvard, « Le petit peuple au Moyen Âge : conclusions », dans P. Boglioni et al. éd., Le Petit Peuple..., p. 707.

33. T. Dutour, « La réhabilitation de l'acteur social en histoire médiévale. Réflexion d'après une expérience de terrain », Genèses, 47 (2002), p. 38. 
doit donc être transitoire, souple et corrigée par d'autres outils afin d'éviter de créer des catégories homogènes, des idéaux types. Il faut en même temps être capable de mesurer la diversité des situations à l'intérieur d'une même catégorie qui regroupe un ensemble de personnages agrégés selon un degré décroissant d'inclusion. Comme à l'intérieur de l'aristocratie il convient de distinguer la haute, la moyenne, la petite aristocratie, en fonction de son origine, son degré de richesse ou sa renommée, de même à l'intérieur du « peuple », une hiérarchie existe entre les mendiants, les «pauvres », les gens de travail, les manouvriers, les domestiques, les gens de métier, les coqs de village, etc. À partir du XII siècle, la reconfiguration des pouvoirs entre seigneurs et communautés villageoises incite certes ces dernières à se penser comme un tout mais, dans le même temps, provoque l'émergence d'une élite villageoise et favorise des hiérarchies au sein du «peuple»: des voix se font davantage entendre que d'autres. Luigi Provero et Laure Verdon insistent à juste titre sur l'hétérogénéité des dominés et écrivent de belles pages sur les représentants et les intermédiaires entre communautés et seigneurs. Longtemps, dans l'historiographie médiévale, la collusion entre « peuple » et « chrétien » par la locution «peuple chrétien », concept de base des représentations sociales du catholicisme, a empêché de penser les différences sociales. Or, il est indispensable d'identifier socialement chaque acteur, car leurs intérêts ne sont pas communs et leurs voix n'ont pas la même intensité. Pour éviter d'être à jamais piégé dans des catégories rigides et une histoire fixiste, en tenant compte des fortes distinctions liées à l'âge, au sexe ou au niveau social, l'historien doit resocialiser les acteurs trop longtemps indifférenciés par les médiévaux et les médiévistes. Ce travail est d'autant plus nécessaire lorsque, comme c'est le cas dans ces trois ouvrages, on étudie les voix et on ausculte une documentation inquisitoire qui a tendance à produire, pour les besoins de l'enquête et la recherche de la vérité, des témoins standardisés. Laure Verdon souligne d'ailleurs combien les voix des témoins finissent par se ressembler, car l'enquête, pour permettre l'établissement juridique de la domination, les transforme en une parole collective grâce aux rituels dans lesquels elles sont produites ${ }^{34}$. Il convient donc de proposer une sociographie des voix : un témoin ne vaut jamais un autre témoin ${ }^{35}$. Sans ce travail préalable, la voix du peuple se distingue mal des autres. Or, on sait par exemple, à y regarder de près, que certains témoins sont toujours davantage habilités à dire la fama publica que d'autres. Comme l'écrit Laure Verdon, « peut-être aussi la fama publica se doit-elle d'être portée par certaines voix plutôt que

34. L. Verdon, La Voix des dominés..., p. 177-178.

35. Pour une histoire sociale des témoins, voir D. LETT, Un procès de canonisation..., p. 185-215 ; et M. Dejoux, Les Enquêtes de Saint Louis. Gouverner er sauver son âme, Paris, 2014, chap. VI, p. 219-272. 
d'autres, celles des notables par exemple, pour acquérir valeur notoire ${ }^{36}$ ? . Dans les procès de canonisation, le peuple, très souvent, n'atteste la fama sanctitatis qu'indirectement, en rapportant des miracles ${ }^{37}$.

Au sein des voix populaires, la distinction possède des limites que la documentation impose. Dans ces trois livres, les voix entendues sont très majoritairement masculines. Bien qu'il n'y ait pas d'interdiction à interroger des femmes en justice, Laure Verdon déplore de ne pouvoir s'appuyer que sur un seul témoignage féminin (et encore, est-ce une nonne) dans les enquêtes provençales visant à prouver la légitimité du droit seigneurial, présent dans le litige opposant Bertrand de Baux à la commune d'Arles, en 1269-1270 ${ }^{38}$. On devine parfois des voix de femmes lors des révoltes. Les trois séditions (en 1355, 1371 et 1388) qui se sont déroulées à Villeveyrac, village du Languedoc, témoignent «d'une très nette implication des femmes », les émeutiers étant, par exemple en 1355, « tam homines quam mulieres ${ }^{39} »$.

\section{Les voix du peuple : de la résistance passive aux révoltes}

Le peuple est saisi ici d'abord en tant que groupe social assujetti (« dominés », «sudditi ») afin de dévoiler les mécanismes de domination et la manière dont se perpétue l'ordre social. Mais les acteurs soumis ne sont pas passifs. Ils refusent ou désobéissent, manifestant une réelle «capacité d'agir »(agency). Pour se faire entendre, le peuple ne s'exprime pas seulement par la violence comme la majorité des chroniqueurs voudrait le laisser croire. Lorsque qu'il juge que les élites oublient de gouverner pour le bien commun et l'utilité publique, il utilise des moyens pour le faire savoir. L'intérêt de ces trois ouvrages est de décrire toute une gamme d'expressions populaires qui participe de la manifestation d'une « économie morale » des plus défavorisés, au sens où Edward $\mathrm{P}$. Thompson l'avait définie ${ }^{40}$. Les formes de luttes des «sans voix » ont d'abord (et sans doute surtout) été silencieuses, comme l'a mis en évidence l'anthropologue James C. Scott en avançant le concept de «transcriptions cachées (hidden transcripts) », largement utilisé dans quelques articles de The Voices of the People ${ }^{41}$. En étudiant la société paysanne d'un village malaisien, James C. Scott a souligné que les modes de résistance s'opèrent le plus souvent de manière passive : sabotages, refus silencieux d'obéir, etc., autant d'actions souterraines qui

36. L. Verdon, La Voix des dominés..., p. 45.

37. D. LetT, Un procès de canonisation..., p. 357-378.

38. L. Verdon, La Voix des dominés..., p. 45, note 68.

39. V. Challet dans The Voices of the People..., p. 133.

40. E. P. Thompson, « The Moral Economy of the English Crowd in the Eighteenth Century », Past \& Present, 50 (1971), p. 76-136.

41. J. C. ScotT, Domination and the Arts of Resistance : Hidden Transcripts, New Haven, 1990. 
aident à créer un esprit de résistance collective de la communauté. Au Moyen Âge, la mauvaise volonté individuelle ou collective des paysans ou les conflits de travail sont déjà une manière efficace d'exprimer sa voix, comme on en trouve de nombreux exemples dans les court rolls anglais ${ }^{42}$. Ensuite, la voix du peuple s'exprime par des moqueries ou des injures à l'égard du pouvoir et, le plus souvent, vis-à-vis de ses représentants locaux (bayles, sergents, etc.). En étudiant les insultes dans les villes de Flandres et de Brabant, Jelle Haemers et Martine Veldhuizen soulignent que les injures sont aussi un moyen populaire efficace de contestation. Patrick Lantschner observe, à partir de cas rapportés dans les sources judiciaires ou des répétitions d'interdiction dans les sources statutaires, des formes de protestations plus insidieuses : déféquer, uriner ou déposer des ordures devant la maison des bourgeois de la ville, sont des formes de résistance («low levels of protest and veiled negotiations $\left.{ }^{43} »\right)$. Le peuple se voit encore offrir des moyens juridiques de contestation par la possibilité de rédiger des pétitions ou d'exprimer des plaintes : lors de l'enquête menée en 1310 dans la baillie de Castellane, les hommes de Biaux accusent les bayles seigneuriaux d'être incompétents et remettent en cause leur nomination ${ }^{44}$; à Venise, s'affrontent représentants de l'autorité publique et habitants de la cité lors des quatre enquêtes instruites entre 1476 et 1519 , étudiées par Claire Judde de La Rivière (The Voices of the People).

Enfin - et l'ouvrage collectif The Voices of the People y consacre une très large place - le peuple s'exprime lors de mouvements plus bruyants : tumultus, rebellio, seditio, rixa, scandalum, etc., termes génériques qui désignent des phénomènes d'ampleur différente. Dans la lignée des travaux de Samuel Cohn, qui a insisté sur le grand nombre de révoltes et leur efficacité à la fin du Moyen Âge ${ }^{45}$, quelques auteurs de The Voices of the People délaissent volontairement le «temps des révoltes 》 (1378-1382) pour se consacrer aux petites séditions ${ }^{46}$. Les causes principales de ces dernières révèlent les préoccupations d'un peuple inquiet : mauvaise récolte, soudure

42. L. Feller, Paysans et seigneurs..., p. 175-176. Voir aussi des exemples de ces résistances paysannes souterraines dans le Jura dans V. CORRIOL, « Redevances symboliques et résistance paysanne au Moyen Âge. À propos du procès de Berthet de Lessart (1423) », Histoire \& Sociétés rurales, 37/1 (2012), p. 15-42.

43. P. Lantschner dans The Voices of the People..., p. 74.

44. L. Verdon, La Voix des dominés..., p. 23.

45. S. CoHn, Lust for Liberty. The Politics of Social Revolt in Medieval Europe, 12001425, Cambridge, 2006.

46. En effet, dans l'historiographie traditionnelle des révoltes "populaires », ont été abondamment étudiées celles des Karls en Flandre maritime dans les années 1324-1328, d'Étienne Marcel et de la jacquerie de 1358, des Tuchins en Languedoc entre 1363 et 1384 et surtout les grandes révoltes des années 1378-1382 : les Ciompi à Florence, les Maillotins à Paris, les « Travailleurs » anglais et la Harelle à Rouen. Pour une bibliographie partielle sur ces révoltes, voir n. 22 ; pour une rapide synthèse, voir C. GAUVARD, « Révoltes populaires », dans C. GAUVARD, A. De Libera et M. ZinK éd., Dictionnaire du Moyen Âge, Paris, 2002, p. 1206-1209. 
difficile, insécurité ou sentiment d'insécurité, volonté d'être autonome en matière de défense ou opposition à une nouvelle taxe. Ces mouvements, qui sont autant des actes que des paroles, ne sont pas aussi désordonnés, violents et sanguinaires que les chroniques se plaisent à les décrire ${ }^{47}$, car les classes subordonnées qui s'insurgent y produisent un langage politique. En étudiant les trois «petites révoltes » qui ont eu lieu à Villeveyrac en Languedoc dans la seconde moitié du XIV ${ }^{\mathrm{e}}$ siècle, Vincent Challet démontre que ces troubles ne concernent pas du tout une minorité mais peuvent enrôler plus de la moitié des adultes d'un village. Pour l'historien, ils sont l'occasion de mesurer l'intensité des voix (le cri, collectif de la foule ou personnalisé), les slogans utilisés, etc. Dans le collectif The Voices of the People, une place très importante (pas moins de trois articles) est faite aux soulèvements armés des villes de Tolède et de Valladolid lors de la guerre des Communautés contre le pouvoir royal en 1520-1522, qui, depuis de nombreuses années, a fait l'objet de virulents débats dans l'historiographie espagnole : simple mouvement antifiscal, révolte antiseigneuriale ou première révolution bourgeoise ? Maria Antonia Carmona Ruiz (en étudiant une chronique, la Relacion de las Communidades de Castilla) ou Hippolito Rafael Oliva Herrer insistent sur l'habilité du peuple à exprimer un discours autonome et politique (« a popular agency»).

\section{Peuple et conscience politique}

Parmi les modes d'expression populaire, une large place est faite, en particulier dans les livres de Laure Verdon et de Luigi Provero, à ceux qui émanent des communautés villageoises face aux seigneurs, dans la lignée des travaux de Chris Wickham ${ }^{48}$. Cet intérêt pour le peuple en tant que groupe social et être politique agissant incite les deux auteurs à centrer leur intérêt sur les formes d'expressions politiques collectives, par l'intermédiaire d'instances de représentation aux dépens des voix individuelles. Si le peuple en tant que groupe peut s'exprimer, produire son propre discours, c'est qu'il possède une culture et un langage politique commun, échangés dans les champs, sur la place du marché, dans l'église paroissiale, dans la boutique ou dans la maison ${ }^{49}$. L'expression d'une voix collective populaire

47. Qu'on pense à la fameuse description de Froissart dans Les Chroniques de France, d'Angleterre et des pais voisins, de la jacquerie survenue en mai-juin 1358 au nord de Paris en lien avec la révolte d'Étienne Marcel. Le chroniqueur cherche par divers moyens à souligner la sauvagerie des paysans qui, « comme des chiens enragés », détruisent les châteaux, tuent les nobles, violent leurs épouses et leurs filles, massacrent les femmes enceintes et contraignent leurs victimes à l'anthropophagie, texte édité dans Historiens et chroniqueurs du Moyen Âge, trad. fr. A. Pauphilet et E. Pognon, Paris, 3e éd., 1986, p. 388-390.

48. C. Wickham, Comunità e clientele...

49. Voir J. WatTs, The Making of Polities (Europe, 1300-1500), Cambridge, 2009. 
et efficace est également bien présente dans les villes, comme le montrent Patrick Lantschner (The Voices of the People), en étudiant les différentes associations de Lille à la fin du Moyen Âge, et Jan Dumolyn (The Voices of the People), en analysant le pouvoir collectif exercé par les artisans des guildes à Gand, Bruges et Ypres au XIV e siècle. Comme les arti dans les communes italiennes, ces guildes ont été un lieu de circulation d'idées politiques et un espace de critique.

Les occasions d'expression d'une conscience politique du peuple sont nombreuses. On les rencontre cependant surtout lors des moments de contestations entre communauté et seigneur : lors des prélèvements, de la levée du cens ou d'une nouvelle taxe, de la délimitation des territoires ou de la redéfinition des droits d'usage. Ces conflits de pouvoir donnent lieu à des rédactions d'écrits : enquêtes, reconnaissance de tenures ou plaintes. Dans un livre important, Thomas N. Bisson avait déjà utilisé seize plaintes (querimoniae) aragonaises de la seconde moitié du XIII ${ }^{\mathrm{e}}$ siècle qui donnaient la parole à des paysans souvent humiliés ${ }^{50}$. Si les princes sont très à l'écoute de la voix de leur peuple par le biais de ces plaintes, c'est qu'ils y voient un intérêt. Ils peuvent se servir en effet des enquêtes de vérité pour régler les conflits les opposant aux seigneurs locaux. Le peuple est donc souvent utilisé comme ressource et sa voix, manipulée. Dans les procédures inquisitoires, devenues, à tous les niveaux, un véritable instrument de pouvoir, les autorités n'hésitent pas à faire appel à la dénonciation, sans mesurer les incidences néfastes de ce procédé sur les communautés : « la parole des témoins, écrit Laure Verdon, sert alors le pouvoir qui ne se préoccupe sans doute pas des conséquences entraînées par la délation sur le tissu social local ${ }^{51}$ ». Dans les procès de canonisation, les paroles «populaires » sont souvent convoquées, contrôlées, utilisées, voire manipulées par et pour les postulateurs de la cause. En ce sens, la procédure inquisitoire qui envahit notre documentation à partir du XIII ${ }^{\mathrm{e}}$ siècle peut être vue comme un instrument supplémentaire de domination sociale entre les mains de l'élite ${ }^{52}$.

L'intérêt d'insister sur cette parole populaire collective et institutionnalisée est de souligner que le peuple ne s'exprime pas, comme les chroniques par exemple le laissent presque toujours supposer, par à-coup, de manière instinctive et violente, comme « un grand enfant », mais que son action s'inscrit dans la durée, se manifeste par une parole ordinaire véhiculée par des instances de représentations, sachant user de stratégies et

50. T. N. Bisson, Tormented Voices. Power, Crisis and Humanity in Rural Catalonia, 1140-1200, Cambridge, 1998.

51. L. Verdon, La Voix des dominés..., p. 178.

52. D. LETT, « La parole des humbles comme ressource. L'utilisation de la procédure inquisitoire par les postulateurs de la cause dans le procès de canonisation de Nicolas de Tolentino (1325) », dans P. Golinelli éd., Agiografia e culture popolari (Hagiography and Popular Cultures), in ricordo di Pietro Boglioni, Bologne, 2012, p. 233-240. 
d'un langage politique élaboré. La voix du peuple fait partie intégrante du fonctionnement du système politique, elle n'a rien d'occasionnelle ou de marginale.

\section{La provenance documentaire des voix}

Parce que les humbles n'ont pratiquement pas laissé de traces écrites de leurs mains, le médiéviste a longtemps pensé que faire leur histoire (comme faire celle des femmes ${ }^{53}$ ou des enfants) ne pouvait être que celle du regard que des hommes adultes portaient sur eux. Aujourd'hui, prenant encore un peu plus ses distances vis-à-vis des dernières scories du positivisme, délaissant l'histoire des représentations, l'historien commence à prendre conscience qu'il construit surtout une histoire du regard qu'il porte sur les acteurs du passé à partir d'un document qu'il transforme en source. Luigi Provero revendique le fait que l'analyse historique passe à travers l'ego de l'historien et l'ego de celui qui a produit le document ${ }^{54}$. Les voix du peuple, comme les voix des élites, en effet, sont médiatisées par les professionnels de l'écrit. Elles ne sont ni plus lointaines ni plus proches de la réalité passée que les autres. Les documents ne sont pas un point de départ, mais le résultat d'un lent processus de production qui doit, lui aussi, être pris en compte et étudié. La «nouvelle érudition » nous a fait comprendre que les médiations notariales agissent de manière puissante sur tous les types de documents ${ }^{55}$. L'historien doit donc remonter à la source et décrypter les nombreux filtres qui se sont sédimentés entre des voix réellement prononcées et leur mise par écrit : interrogatoire, contrainte d'énonciation, contexte interlocutoire, passage de l'oral à l'écrit, du vulgaire au latin, etc. ${ }^{56}$ Nous ne sommes plus au temps du projet utopique, développé par l'anthropologie historique, de l'historien se faisant ethnologue des sociétés du passé, comme si notre documentation permettait d'accéder à la voix vive de ceux et celles qui avaient parlé ${ }^{57}$. Pour l'historien, en particulier celui qui travaille sur des

53. Il suffit, pour s'en convaincre, de relire les préfaces des premiers volumes de G. Duby et M. Perrot (éd.), Histoire des femmes en Occident, Paris, 1991.

54. L. Provero, Le Parole dei sudditti..., p. Xvi.

55. Voir, en particulier, É. Anheim et P. Chastang éd., Les Pratiques de l'écrit dans les sociétés médiévales (VI'-XIII siècle), dossier paru dans Médiévales, 56 (2009).

56. D. LETT, « La langue du témoin sous la plume du notaire : témoignages oraux et rédaction de procès de canonisation au début du XIv ${ }^{\mathrm{e}}$ siècle » dans L'Autorité de l'écrit au Moyen Âge (OrientOccident), XXXI Congrès de la SHMESP, Le Caire, 30 avril-5 mai 2008, Paris, 2009, p. 89-105.

57. Ainsi Michael Goodich pense que les sources inquisitoires, grâce à la procédure notariale, «nous permet[tent] souvent d'apercevoir les voix authentiques d'un large spectre de participants et témoins de miracle » : M. Goodich, Violence and Miracle in the Fourteenth Century. Private Grief and Public Salvation, Chicago, 1995, p. 9. C'est aussi ce que pense Stanko Andric, en citant cette phrase de Michael Goodich : S. Andric, The Miracles of St. John Capistran, Budapest, 2000, p. 228. Paolo Mariani déclare également, qu'avec les 
sources inquisitoires qui semblent donner la parole aux témoins, un écueil grave a été en effet de " prendre les interrogatoires d'inquisiteurs pour des enquêtes ethnographiques ${ }^{58} »$ :

Lorsqu'ils considèrent ces témoignages comme des procès-verbaux de gendarmerie [...] ou des comptes rendus de séance parlementaire, les historiens se transforment, sans s'en apercevoir, en fées fileuses de mythes ${ }^{59}$.

Même si la procédure a besoin de ce récit oral premier, elle a «fait taire définitivement » la voix vive «en imposant l'écrit » ${ }^{60}$. Entendre la voix du peuple à travers des sources écrites, s'intéresser au caractère oral de la langue est aussi un phénomène relativement récent dans la linguistique actuelle ${ }^{61}$, la philologie traditionnelle ayant largement privilégié les sources littéraires « hautes » donc « écrites », d'où un certain mépris pour les sources « basses » (telle la farce par exemple) qualifiées parfois, de manière péjorative, de folklore.

Les deux livres et les articles retenus ici portent donc une réflexion aiguë sur les mécanismes qui ont produit cette documentation permettant à ces voix de résonner jusqu'à nous, dégradées, atténuées, amoindries. Dans ces positionnements théoriques, explicitement ou non, on devine l'influence de nouveaux paradigmes : certains apports du linguisitic turn ${ }^{62}$, qui attirent l'attention sur les liens entre réalité historique et médiation du langage, de l'anthropologie critique qui autorisent à des réflexions plus soutenues sur les pratiques de communication, les procédés d'énonciation et le contexte interlocutoire $^{63}$ (notion de perlocutory speech utilisée dans The Voices of the People), de la nouvelle érudition ${ }^{64}$, des subaltern studies qui dirigent le regard vers les subaltern spaces dans lesquels le peuple s'exprime bien davantage que dans les lieux officiels du pouvoir ${ }^{65}$, ou encore de la microstoria, clairement revendiqués par exemple par Luigi Provero en introduction de

procès de canonisation, « nous avons à notre disposition la "voix-vive" d'un "quasi-muet" », c'est-à-dire celle du peuple : P. MARIANI, « Racconto spontaneo o memoria costruita ? Testi a confronto in alcuni processi di canonizzazione del secolo decimoquarto », Mélanges de l'École française de Rome - Moyen Âge, 108/1 (1996), p. 259-319 (p. 260).

58. J. ChIFFOleAu, « Dire l'indicible. Remarques sur la catégorie du nefandum du XII au XV ${ }^{\mathrm{e}}$ siècle », Annales ESC, 45/2 (1990), p. 309.

59. J. FRIED, « Le passé à la merci de l'oralité et du souvenir. Le baptême de Clovis et la vie de Benoît de Nursie », dans J.-C. Schmitt et O. G. Exle éd., Les Tendances actuelles..., p. 72.

60. J. ChIFFoleau, « Dire l'indicible... », p. 309.

61. Dans la lignée des travaux de P. ZumThor, La Lettre et la Voix. De la «littérature » médiévale, Paris, 1987.

62. Revendiqué dans l'introduction de J. Dumolyn et al.éd., The Voices of the People..., p. 6.

63. Pour les travaux d'anthropologie critique sur l'interlocution qui, en particulier, remettent en cause la passivité du « subordonné » face à l'ethnologue, voir B. MASQUELIER et J. L. Siran éd., Pour une anthropologie de l'interlocution. Rhétoriques du quotidien, Paris, 2000 ; et É. Chauvier, Anthropologie de l'ordinaire. Une conversion du regard, Paris, 2011.

64. Voir les références bibliographiques citées n. 45.

65. Voir l'article de H. R. Oliva Herrer dans The Voices of the People..., p. 50. 
son ouvrage ${ }^{66}$, qui ne présuppose pas un contexte mais qui souhaite partir d'abord des flux documentaires. La réduction d'échelle permet aujourd'hui assurément de mieux être à l'écoute des gens ordinaires, paradoxe qui voit l'historien délaisser les masses pour se focaliser sur les gens du peuple.

Dans ces trois ouvrages, de nombreuses sources sont convoquées. On l'aura compris, l'enquête est largement privilégiée car elle rapporte des traces écrites de paroles prononcées par des témoins ${ }^{67}$. Laure Verdon cherche à saisir l'opinion des dominés face à la manifestation du pouvoir en s'appuyant principalement sur les enquêtes qui se multiplient en Provence à partir de l'affirmation du pouvoir angevin au XIII ${ }^{\mathrm{e}}$ siècle $^{68}$ et des reconnaissances de tenure. C'est pourquoi, elle consacre son premier chapitre (qui remplace l'introduction) à une réflexion méthodologique et historiographique sur l'enquête et l'inquisitoire. Sont aussi largement utilisées les chartes de franchises, concessions seigneuriales qui rendent « libre » une communauté d'un ensemble d'obligations et qui redéfinissent les relations entre la communauté et le seigneur. La charte de franchise n'est en effet jamais le fruit d'une pure imposition, même si elle oppose deux parties de force inégales. En les scrutant attentivement, on peut reconstituer, sur tous les principaux points de conflits potentiels, des bribes de voix du peuple à propos de la recherche de compromis autour de la procédure judiciaire, les biens communs, les prélèvements, la défense du village, etc., comme le fait Luigi Provero à partir d'un corpus de deux cents actes environs des $\mathrm{XII}^{\mathrm{e}}$-XIII ${ }^{\mathrm{e}}$ siècle du Piémont méridional. Benoît Cursente avait déjà proposé de retrouver la voix des simples paysans à travers ce type de documentation ${ }^{69}$. Les propositions faites par Luigi Provero permettent de sortir de l'opposition classique et rigide entre, d'un côté, ceux qui voient les chartes de franchises comme un succès des communautés qui obtiennent des concessions et, de l'autre, ceux qui les perçoivent comme un acte des seigneurs, servant à consolider leur propre pouvoir. Il propose une troisième voie. Selon lui, les franchises sont « uno strumento della politica locale, un utensile che permette a tutti i gruppi politicamente attivi di modellare

66. L. Provero, Le Parole dei sudditti..., p. xv.

67. C. Gauvard éd., L'Enquête au Moyen Âge, Rome, 2008.

68. Laure Verdon inscrit son étude au sein du travail collectif mené depuis plusieurs années, sous la direction de Thierry Pécout, sur l'enquête générale effectuée dans l'ensemble de la Provence angevine sur ordre de Robert Ier entre 1331 et 1334 . Voir T. PÉcout, « Du castrum au registre et vice-versa. L'enquête générale en Provence angevine, fin XIII ${ }^{\mathrm{e}}$-fin XIV ${ }^{\mathrm{e}}$ siècle », Rives méditerranéennes, 37 (2010); et l'édition des enquêtes pour la partie orientale de la Provence, T. PÉCOUT éd., L’Enquête générale de Leopardo da Foligno en Provence orientale (avril-juin 1333), Paris, 2008.

69. B. Cursente, «Franchises et prélèvements dans la France des XII $-\mathrm{XIII}^{\mathrm{e}}$ siècles : la lettre des chartes et la voix des paysans », dans M. Bourin et P. Martinez Sopena éd., Pour une anthropologie du prélèvement seigneurial dans les campagnes (XI -XIV siècles). Réalités et représentations paysannes, Paris, 2004, p. 115-132. 
le forme della convivenza organizzata ${ }^{70} \gg$. La rédaction d'une franchise ne signifie donc ni une accentuation du pouvoir seigneurial ni une efficace résistance de la communauté, mais plus simplement une phase d'intense interaction politique entre les parties.

Autre source convoquée ici pour y chercher la voix du peuple : les statuts communaux, les coutumes, les ordonnances ou les codes de lois. Cette documentation, pourtant très bien connue et exploitée, a trop longtemps été perçue comme une source normative, appréhendée sur un plan juridique. Or, on doit l'étudier autrement et la considérer également comme une source de la pratique ${ }^{71}$. La matière statutaire, en effet, centrée sur des usages locaux, profondément ancrée dans un territoire, remaniée sans cesse (correctiones, additiones visibles dans les marges ou en fin de manuscrits), rédigée par des statutarii, certes épaulés par des spécialistes du droit mais, très souvent, dans les petites communautés, des gens du peuple fortement impliqués dans les affaires locales et la vie publique, donne aussi accès à une forme de connaissance des pratiques. En utilisant une analyse lexicale, Jonas Braekevelt (The Voices of the People) met au jour des formes de négociation et une voix populaire à travers les ordonnances princières flamandes sous Philippe le Bon qui parfois recopient des requêtes élaborées par les sujets.

D'autres types de documents sont encore utilisés de manière originale. Ainsi Frances Andrews (The Voices of the People) étudie un sermon de frère Venturino de Bergame prononcé à Rome en 1335 pour démontrer qu'on ne peut le comprendre sans une réflexion sur ceux qui l'écoutent. Le peuple est aussi celui qui reçoit une parole. Le prédicateur doit parfaitement connaitre son auditoire (on connaît la performativité des sermons ad status) pour rendre sa parole efficace. À partir des sermons, on peut donc reconstruire la performance orale du prédicateur et percevoir les gens du peuple en tant que listeners.

La liste des documents à utiliser pour entendre la voix du peuple n'est pas close et les trois ouvrages n'avaient pas vocation à l'épuiser. Les catasti ou estimi italiens, par exemple, demeurent toujours une source très riche où les paroles des gens ordinaires surgissent, où « comme à leur habitude, les contribuables font preuve d'une loquacité qui ne leur était pas demandée $^{72} \gg$. On se souvient de cet humble métayer florentin, Bernardo

70. L. Provero, Le Parole dei sudditti..., p. 11.

71. Nous développons cette idée au sein d'un programme de recherche (2012-2016) que je dirige à l'École française de Rome (Axe 4, thème 12), intitulé « Statuts, écritures et pratiques sociales dans les sociétés de la Méditerranée occidentale à la fin du Moyen Âge (XII$\mathrm{XV}^{\mathrm{e}}$ siècle) », [en ligne] : http://www.ecole-francaise.it/fr/q/d/Programme_4.12.2.pdf [consulté le 7 septembre 2016] Sur le rapport entre normes et pratiques, voir la judicieuse mise au point de S. CerutTI, « Normes et pratiques, ou de la légitimité de leur opposition », dans B. LePETIT éd., Les Formes de l'expérience. Une autre histoire sociale, Paris, 1995, p. 127-149.

72. M. Scherman, Familles et travail à Trévise à la fin du Moyen Âge (vers 1434vers 1509), Rome, 2013, p. 76. 
Lanciani, qui, en 1469, explique aux employés du fisc que son premier fils Antonio est mort et qu'il l'a « refait» $(\text { Mori e refecilo })^{73}$. Dans ce type de déclaration, les plus pauvres usent parfois d'ironie tel ce déclarant de Trévise, Andrea quodam Girardo da Valmareno qui précise en 1447 qu'il est tellement riche qu'il est obligé de mendier, ou cet autre, en 1455, barbier, maître Jacomo dit Botarel de Florence, qui, pour exprimer la valeur de son patrimoine, commence sa phrase par «Item ho.... » et ajoute vingtquatre zéros ${ }^{74}$. Dans les registres de délibérations communales italiens, on rencontre aussi de très nombreuses pétitions de pauvres gens, vieux, handicapés, femmes délaissées ou orphelins, venant quémander auprès des autorités un dégrèvement ou une annulation d'impôts : à Macerata, de nombreuses veuves (uxor olim ou uxor quondam) viennent exprimer leur indigence et leur souffrance dans les petitiones rapportées dans une riformanza de 1432, telle cette «Lucia, épouse de feu Jacopo di Bartolomeo, veuve et misérable femme », qui déclare ne rien posséder, " même pas une petite maison et à qui il reste de son mari deux petites filles très pauvres ${ }^{75}$ ». Écouter les voix du peuple, c'est aussi être attentif à la charge émotionnelle de ces paroles, comme le souligne Laure Verdon à la fin de son ouvrage ${ }^{76}$ en s'appuyant sur les travaux de Sophie Wahnich, de Barbara H. Roseinwein et de Damien Boquet et Piroska Nagy ${ }^{77}$. Les insultes échangées entre habitants, rapportées dans les libelles des registres de justice des communes italiennes (libri maleficiorum), sont encore une mine pour écouter la voix du vulgus. On fera remarquer, pour terminer, que petitiones et injures sont le plus souvent rédigées en langue vernaculaire au sein d'un texte latin, ce qui impose à l'historien de s'interroger davantage qu'il n'est fait dans ces trois ouvrages sur la langue de la voix du peuple et sur l'articulation entre le latin et le « vulgaire ». Seul Vincent Challet évoque la langue dans laquelle s'est exprimée la voix des insurgés ${ }^{78}$. Car il faut aussi se demander pourquoi le notaire, dans certains contextes documentaires, ne traduit pas dans la lingua sacra certaines paroles «populaires ». Pour donner un « effet de réel »

73. C. Klapisch Zuber, «Le nom "refait". La transmission des prénoms à Florence (XIve$\mathrm{XVI}^{\mathrm{e}}$ siècles) », L'Homme, 20/4 (1980), p. 77-104, repris dans EAD., La Maison et le nom. Stratégies et rituels dans l'Italie de la Renaissance, Paris, 1990, p. 83-107 (p. 99).

74. M. Scherman, Familles et travail..., p. 51. À partir de neuf registres fiscaux (estimi) rédigés entre 1434 et 1499, riches de plus de 7000 déclarations, Matthieu Scherman peut « écouter » près de 14000 contribuables.

75. Macerata, Archivio di Stato, Riformanza 15 (1432), f. 99.

76. L. VERDON, La Voix des dominés..., p. 171-176: « La place de l'affect ».

77. S. WAHNich, Les Émotions. La Révolution française et le présent. Exercices pratiques de conscience historique, Paris, 2009 ; B. Rosenwern, Emotionnal Communities in the Early Middle Ages, Ithaca/Londres, 2006 ; D. Boquet et P. NAGY éd., Le Sujet des émotions au Moyen Âge, Paris, 2008 ; EID. éd., Politiques des émotions au Moyen Âge, Florence, 2010 ; voir aussi désormais EID., Sensible Moyen Âge. Une histoire des émotions dans l'Occident médiéval, Paris, 2015.

78. V. Challet dans The Voices of the People..., p. 136. 
supplémentaire ? Pour prendre des distances à l'égard d'injures dégradantes (putana, merda, etc.), de blasphèmes («Per la porta della virgine Maria») ou de cris de révoltes («Mueran, mueran ») ? La parole du peuple seraitelle plus vulgaire qu'une autre ${ }^{79}$ ?

Le peuple est une production historiographique de l'Ecclesia («peuple chrétien ») et du XIX ${ }^{e}$ siècle (Michelet), au centre de la culture politique depuis la Révolution française. Ce double héritage en a fait une catégorie unique, un tout, qui a été longtemps (ou est encore) un obstacle épistémologique à une pensée de la différence sociale. Ces trois ouvrages nous aident, chacun à leur manière, à déconstruire cette catégorie. Malgré les titres (dominés, suditti), les ouvrages de Laure Verdon et de Luigi Provero dépassent l'opposition simpliste entre dominants et dominés en montrant la multiplicité des protagonistes qui participent aux négociations politiques entre seigneurs et communautés rurales et les voix discordantes à l'intérieur des gens du peuple. The Voices of the People offre toute une gamme d'expressions populaires performatives car « dire c'est fair ${ }^{80} »$. Les historiens du social ont été trop longtemps indifférents aux différences. Si les médiévistes veulent vraiment mettre au jour la société dans son intégralité et sa discontinuité, avec ses émotions et ses inégalités sociales, la moindre des choses est de rendre la parole à l'écrasante majorité des acteurs de l'histoire aux « sans voix » qui, grâce à ces trois ouvrages, sont un peu moins des « gens sans archives ni visages ${ }^{81} »$.

Didier Lett - Université Paris 7 - Paris Diderot

79. Voir Y. CaZal, Les Voix du peuple. Verbum Dei. Le bilinguisme latin-langue vulgaire au Moyen Âge, Paris, 1998, qui étudie les intrusions du vulgaire dans les textes latins, les farcitudes et les drames bilingues.

80. J. L. Austin, Quand dire, c'est faire, Paris, [1962] 1970.

81. A. J. Gourevitch, La Culture populaire ..., p. 10-11. 